\title{
Classical dynamical modelling of near-barrier breakup
}

\author{
E. C. Simpson ${ }^{1, \star}$, K. J. Cook ${ }^{1}$, Sunil Kalkal ${ }^{1}$, D. H. Luong ${ }^{1}$, I. P. Carter ${ }^{1}$, M. Dasgupta ${ }^{1}$, and D. J. Hinde ${ }^{1}$, \\ ${ }^{1}$ Department of Nuclear Physics, Research School of Physics and Engineering, Australian National University, Canberra, ACT \\ 2601 Australia
}

\begin{abstract}
The complete fusion of light, weakly-bound nuclides is known to be significantly suppressed with respect to comparable well-bound projectiles or with respect to single barrier penetration model calculations. Strong $\alpha$-clustering in these light systems mean that they very easily disintegrate into clusters, either via direct excitation of their intrinsic cluster continuum, or via transfer reactions which connect to unbound states in neighbouring nuclides. This breakup is thought to reduce the probability for complete fusion. Here we discuss which processes cause breakup, whether or not breakup happens fast enough, and the interpretation of measurements made at the Australian National University of breakup using classical dynamical models. Understanding the intimate details of breakup, and the resonances through which it proceeds, will be crucial in determining its likely influence on fusion.
\end{abstract}

\section{Introduction}

Heavy-ion fusion is necessarily a many-body process. It involves dissipation of the collision kinetic energy into a multitude of internal degrees of freedom, in order to form a single compound system. It is an ideal testing ground for quantum dynamics and many-body tunnelling. Despite this, couplings to low energy collective states, or transfer of individual nucleons between the projectile and target, can have profound effects on fusion cross sections [1]. To understand fusion, it is crucial to understand the interplay between these low excitation, few-body degrees of freedom, and the complex dissipative effects ultimately required for fusion.

Few-body effects are particularly prevalent in nearbarrier reactions of light weakly-bound nuclides. The strong binding of the $\alpha$-particle makes nuclides with $A<$ 10 intrinsically fragile. The lowest cluster separation thresholds for the stable nuclides ${ }^{6} \mathrm{Li},{ }^{7} \mathrm{Li}$ and ${ }^{9} \mathrm{Be}$ are all less than 2.5 MeV [2]. This makes them liable to breakup in nuclear collisions, which in turn is believed to have importance consequences for fusion. Indeed, above barrier complete fusion of light, weakly-bound projectiles with heavy targets is known to be suppressed by $25-35 \%$, when compared to either coupled channels calculations or comparable examples with well-bound projectiles $[3,4]$. It is thought that breakup processes reduce the probability for complete capture of the projectile's charge. A reduction of the complete fusion cross sections is associated with increased incomplete fusion - usually interpreted as capture of one of the breakup fragments following breakup.

Exactly how and why fusion is suppressed in these nuclides is a topic of very active research (see Ref. [5] for

\footnotetext{
^e-mail: edward.simpson@anu.edu.au
}

a recent review). FUSION17 encompassed a large number of talks addressing different aspects of the reactions of these light weakly-bound nuclides, including measurements of fusion and reaction cross sections (Aguilera [6]), quasielastic scattering (Mukherjee), charged-particle conincidences (Kalkal [7], Cook [8], G. L. Zhang [9]), and attempts to model incomplete fusion (Parkar [10]).

As we progress towards more exotic nuclides, understanding the consequences of weak-binding on fusion becomes increasingly important. At this conference seen talks experimental measurements of reactions of ${ }^{7} \mathrm{Be}$ (Mazzocco), ${ }^{8} \mathrm{~B}$ (Kolata [11]), and the halo nucleus ${ }^{11} \mathrm{Be}$ (Pesudo [12]), in addition to time-dependent Hartree-Fock calculations for exotic calcium isotopes (Vo-Phouc [13]). Innovative technical developments were also presented, such as new methods for extraction of fusion excitation functions with stacked targets, which will soon be utilized in experiments with exotic beams (Di Pietro [14, 15]).

What has emerged from recent works with stable beams is that the breakup mechanisms of these nuclides are complex and diverse [16-18]. Here we discuss these reactions, not aiming to quantify to what degree fusion is suppressed, but to understand the manner in which breakup could suppress fusion. We consider sub-barrier breakup reactions, where absorption of the breakup fragments through incomplete fusion is (nearly) eliminated, allowing us to focus exclusively on breakup. In Section 2 we discuss the mechanisms which cause breakup, before considering whether or not breakup happens fast enough to influence fusion in Section 3. Then in Section 4 we discuss classical dynamical models of breakup, which can provide further insights into the detail and timescales of breakup. In Section 5 we discuss these results and the out- 


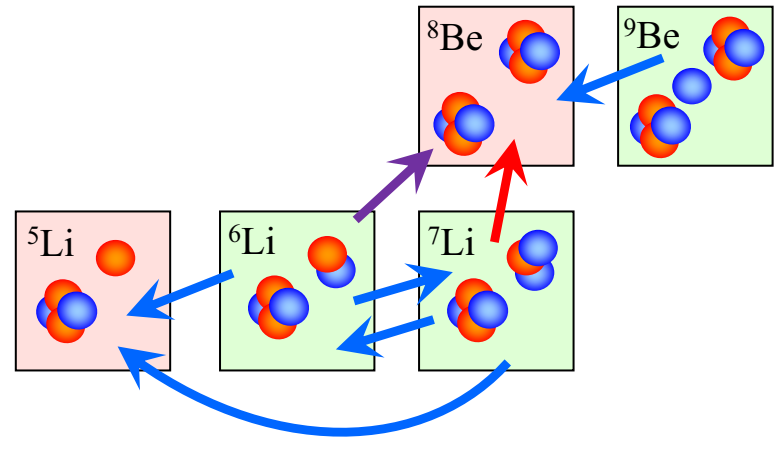

Figure 1. (Colour online) Diagram illustrating the possible transfers that lead to breakup in the reactions of ${ }^{6} \mathrm{Li},{ }^{7} \mathrm{Li}$ and ${ }^{9} \mathrm{Be}$. Blue lines indicate neutron transfers, red lines indicate proton transfers, and the purple line indicates $n p$ transfer. ${ }^{5} \mathrm{Li}$ and ${ }^{8} \mathrm{Be}$ are both unbound, and though ${ }^{6,7} \mathrm{Li}$ and ${ }^{9} \mathrm{Be}$ are bound, their cluster breakup thresholds are low.

look for classical dynamical models and investigations of fusion suppression.

\section{What processes cause breakup?}

The first question we must ask is what mechanism causes light weakly-bound nuclides break up? The strong binding of the $\alpha$-particle means that light nuclides with $A<10$ have low cluster breakup thresholds: ${ }^{6} \mathrm{Li} \rightarrow \alpha+d$ at 1.474 $\mathrm{MeV},{ }^{7} \mathrm{Li} \rightarrow \alpha+t$ at $2.468 \mathrm{MeV}$, and ${ }^{9} \mathrm{Be} \rightarrow \alpha+\alpha+n$ at $1.665 \mathrm{MeV}$. Direct excitation of the intrinsic cluster continuum of the projectile is one possible trigger of breakup. Indeed, many works have noted a correlation between the direct breakup threshold and the degree of fusion suppression $[19,20]$. This correlation is apparent not only for weakly-bound stable nuclides such as ${ }^{6,7} \mathrm{Li},{ }^{9} \mathrm{Be}$ and ${ }^{6} \mathrm{He}$, but also for more well-bound nuclides such as ${ }^{10,11} \mathrm{~B},{ }^{12,13} \mathrm{C}$ and ${ }^{16} \mathrm{O}$. So on the face of it we might conclude that direct breakup causes fusion suppression.

The reality is not quite so simple. Whilst direct excitation of the intrinsic cluster continua does contribute to breakup, strong $\alpha$-clustering in this region means that many neighbouring nuclides are also very weakly bound or unbound, and transfer reactions can connect these different cluster states. This is illustrated in Figure 1 for ${ }^{6} \mathrm{Li}$, ${ }^{7} \mathrm{Li}$ and ${ }^{9} \mathrm{Be}$. For ${ }^{7} \mathrm{Li}$ projectiles, neutron stripping can give unbound states in ${ }^{6} \mathrm{Li}$ resulting in $\alpha+d$, two-neutron stripping gives unbound ${ }^{5} \mathrm{Li}$ and results in $\alpha+p$, and proton pickup gives unbound ${ }^{8} \mathrm{Be}$ and $\alpha+\alpha$. Similar transferinduced breakup modes exist for ${ }^{6} \mathrm{Li}$ and ${ }^{9} \mathrm{Be}$.

Recent measurements of charged particle coincidences have revealed that these transfer-induced breakup modes are indeed important for ${ }^{6,7} \mathrm{Li}$ and ${ }^{9} \mathrm{Be}$ [16-18]. The fractions for the different breakup modes are illustrated in Figure 2. These results include an efficiency correction which depends on the breakup mode, and though this efficiency correction was improved upon in later work (see Ref. [8], we do not anticipate that this change will significantly alter the deduced breakup fractions. It should also be noted that the fractions shown exclude breakup from long-lived
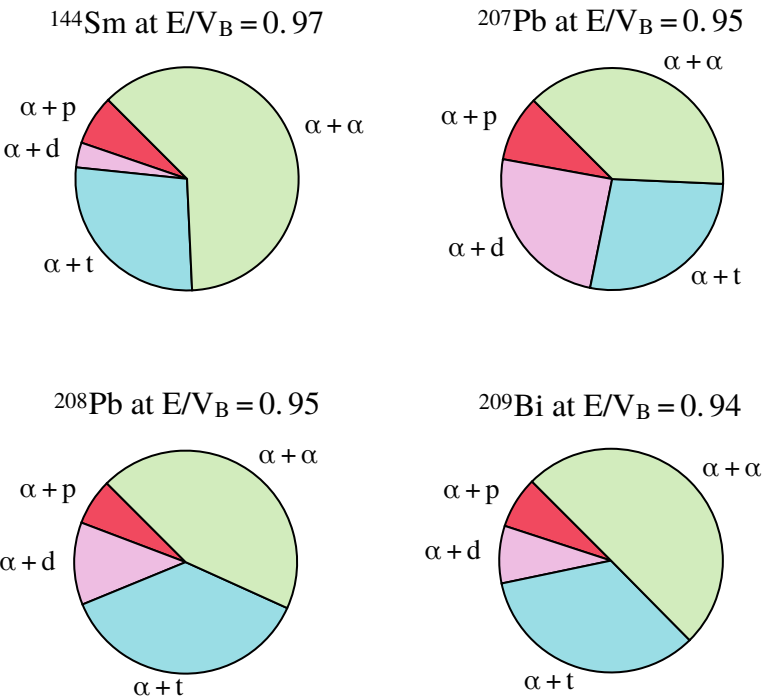

Figure 2. Fractional contribution to breakup for interactions of ${ }^{7} \mathrm{Li}$ with ${ }^{144} \mathrm{Sm},{ }^{207} \mathrm{~Pb},{ }^{208} \mathrm{~Pb}$ and ${ }^{209} \mathrm{Bi}$ [18]. The modes shown are direct breakup (teal), proton pickup (green), neutron stripping (pink) and two-neutron stripping (red). Note that the effect of narrow resonaces has been excluded - see the text for further details.

resonances, as only prompt breakup is expected to result in fusion suppression. In all cases direct breakup into $\alpha+t$ is significant, but the strongest prompt breakup mode is proton pickup to produce $\alpha+\alpha$. As the energy increases towards the barrier energy, the fractional contribution of these transfer-induced breakup modes tends to increase [18].

Whilst the fraction of direct breakup is reasonably constant across the targets presented in Figure 2, the relative strengths of the transfer-induced breakup mechanisms vary markedly. Significant differences occur even when the target is changed by just a single nucleon, driven by changes in transfer $Q$-values. For lighter targets, where the Coulomb field is much smaller, direct breakup is found to be considerably reduced for ${ }^{6} \mathrm{Li}$, and negligible for ${ }^{7} \mathrm{Li}$ [7].

\section{Does breakup happen fast enough?}

Knowing the relevant breakup mechanism is, however, insufficient. In addition to know how breakup occurs, we must also consider whether it occurs fast enough to be able to influence fusion. In a classical sense, if breakup is to have a chance of suppressing fusion, the distinct clusters must be produced before the projectile-like object reaches the fusion barrier at $R_{B}$. If breakup occurs only once the projectile (or projectile-like reaction product) has passed inside the barrier, it is unlikely that either breakup fragment will escape fusion with the target, effectively resulting in complete fusion.

Here our focus will be on sub-barrier breakup measurements. At sub-barrier energies, the classical trajectory of the projectile and target never reaches the fusion barrier at $R_{B}$. As a result, the probability of fusion of the fragments after breakup (i.e. incomplete fusion) is neg- 
(a) Breakup
before $\mathrm{R}_{\mathrm{B}}$

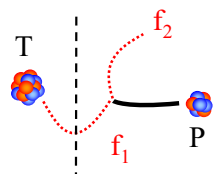

(b)

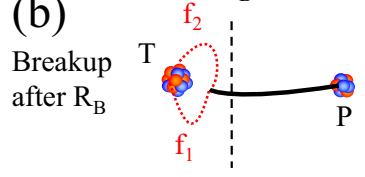

(c)

Breakup before $\mathrm{R}_{0}$

Breakup after $\mathrm{R}_{0}$

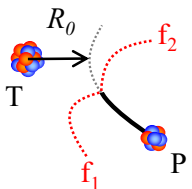

$f_{1}$

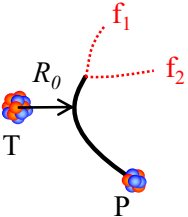

Figure 3. Illustration of breakup at above-barrier energies [(a) and (b)] and sub-barrier energies [(c) and (d)]. (a) At abovebarrier energies breakup prior to the projectile reaching the fusion barrier has the capacity to suppress fusion, whilst (b) breakup once the projectile has already passed inside $R_{B}$ cannot. (c) At sub-barrier energies breakup prior to $R_{B}$ [i.e. (a)] corresponds to breakup prior to the distance of closest approach, whereas (d) breakup after $R_{B}$ corresponds to breakup after the distance of closest approach has been passed.

ligible, allowing the clearest possible picture of breakup to be obtained. To affect fusion at above-barrier energies, breakup must occur prior to $R_{B}$. This loosely translates, at sub-barrier energies, into breakup occurring when the projectile is still approaching the distance of closest approach $R_{0}$. This is illustrated in Figure 3 .

Given the variety of different mechanisms that can trigger breakup, it is necessary to consider not only the structure of the light nuclides in question $\left({ }^{6} \mathrm{Li},{ }^{7} \mathrm{Li}\right.$ and $\left.{ }^{9} \mathrm{Be}\right)$, but also their close neighbours $\left({ }^{5} \mathrm{Li}\right.$ and $\left.{ }^{8} \mathrm{Be}\right)$. This is illustrated in Figure 4. In each case, known bound states and resonances are plotted relative to the lowest breakup threshold. Unbound states, found in the pink shaded section are marked by a black line and, if their width is substantial, a shaded red region indicates their approximate width. The density of resonant states is low, but it is possible also that non-resonant partial waves may contribute to breakup. The relative importance of different resonant states for a given breakup mode, may also depend on the mechanism $-\alpha+d$ produced via direct breakup of ${ }^{6} \mathrm{Li}$ may not be the same as $\alpha+d$ produced via neutron stripping from ${ }^{7} \mathrm{Li}$.

What has emerged from recent work is that the structure of these nuclides is, in fact, crucial. For example, suppose the breakup occurs via a (moderately) narrow resonant state, such as the $3^{+}$resonance in ${ }^{6} \mathrm{Li}$. This state, with a width of $24 \mathrm{keV}$, has a mean lifetime of $27 \times 10^{-20}$ $\mathrm{s}$, more than an order of magnitude longer than the interaction timescale. In an above-barrier collision, it is unlikely to break into fragments (in this case $\alpha+d$ ) before reaching the fusion barrier. This breakup is referred to as asymptotic since, at sub-barrier energies, narrow resonances will only decay into fragments once they have travelled far from the target. This cannot cause fusion suppression. Indeed, if the state is quasi-bound in this sense, it should act as any other coherent inelastic coupling. We are therefore interested in identifying breakup that occurs promptly, on the timescale of the close interaction of the projectile and

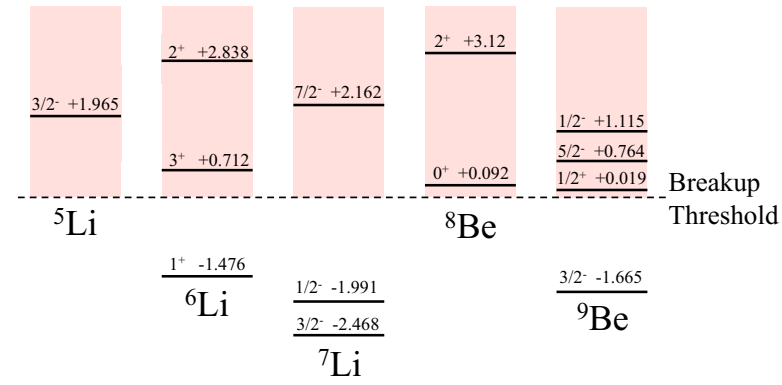

Figure 4. Sketch of the structure of light nuclei relative to their lowest breakup threshold. Horizontal black lines indicate known bound states and resonances. For resonances where the width is substantial, the approximate width is indicated by the red shaded region around the state.

target. Prompt breakup may proceed through these wide resonant states, or via non-resonant parts of the continuum - for example, in the case of ${ }^{6} \mathrm{Li}$, where there are no broad resonances.

One way to experimentally distinguish prompt and asymptotic breakup is via the relative energy distribution $E_{r e l}$ of the two breakup fragments. As asymptotic breakup occurs far from the target, the interaction of the fragments and target is weak, and their relative energy remains characteristic of the state in question and can be cleanly gated out. This was the method used in Ref. [18] to exclude asymptotic breakup from the fractions given in Figure 2. We emphasize that identifying and excluding asymptotic breakup fundamentally requires coincidence measurements - singles measurements cannot help here.

Separating prompt and asymptotic breakup is, however, insufficient. As discussed earlier, at sub-barrier energies we wish to distinguish prompt breakup occurring before and after the distance of closest approach. Prompt breakup that occurs after the projectile is receding from the target corresponds to breakup after $R_{B}$ and will thus not contribute to fusion suppression.

\section{Classical dynamical models of breakup}

The complexity of the reactions of ${ }^{6} \mathrm{Li},{ }^{7} \mathrm{Li}$ and ${ }^{9} \mathrm{Be}$ make them difficult to treat in a complete fashion (i.e. including all channels) with quantum models. Not only must we treat nucleon transfer, but also the coupling between different continuum states of the resulting breakup product. Furthermore, we cannot describe incomplete fusion with such models - fusion, treated using an absorptive potential, makes no distinction between complete and incomplete fusion.

One alternative approach is to use stochastic classical dynamical models [21]. In the model, the classical trajectories of the projectile and target are propagated in time, treating the breakup stochastically. The trajectories of the fragments and target-like reaction product are tracked, allowing the energy and angle correlations of the breakup fragments to be determined, and the cross sections for incomplete fusion to be estimated. Due to some important 
physical and technical limitations of existing implementations of the model [22], a new code has been written. Here we give a brief overview of the model and recent developments of it, and discuss recent results.

\subsection{Recent developments}

The initial condition for each simulation is set by stochastically sampling an impact parameter $b$ below some user defined maximum $b_{\max }$. This differs from the previous implementation [22], where a quantized angular momentum $L$ was sampled. The use of an impact parameter $b$ is more consistent with the classical nature of the model. The projectile and target trajectories are then tracked, assuming their interaction is described with a Coulomb plus WoodsSaxon nuclear potential. The parameters of these potentials are usually constrained to fit the Sao Paulo potential [23], or, in the case of the breakup fragments, light-ion scattering (e.g., $\alpha+\alpha$ ).

The reaction causing breakup is treated as a stochastic process, with the probability of the reaction assumed to depend exponentially on the distance between the projectile and target. The details of this exponential dependence (the slope and normalisation) are determined to match the experimentally measured breakup function, which is just the breakup probability as a function of distance of closet approach $[18,24]$. At the point of reaction, a distribution of excitations $E_{x}$ of the projectile-like nucleus, and corresponding lifetimes $\tau$ for the intermediate nucleus (e.g., ${ }^{8} \mathrm{Be}$ ), are randomly sampled. In the case of proton pickup by ${ }^{7} \mathrm{Li}$, the lifetime represents how long it takes the ${ }^{8} \mathrm{Be}^{*}$ resonance state to decay into two $\alpha$ particles. In the case of the ${ }^{8} \mathrm{Be}$ ground state this time is long - of the order of $10^{-16} \mathrm{~s}$ - but it is much shorter in the case of the ${ }^{8} \mathrm{Be} 2^{+}$. Here, these distributions are described using a one-level one-channel limit of R-matrix theory [25, 26]. This is an important improvement over Ref. [22], which assumed either a flat or exponential distribution with $E_{x}$, and did not explicitly account for the lifetimes of the intermediate nucleus.

The positions of the projectile-like and target-like nuclei are then propagated until the randomly sampled lifetime $\tau$ has elapsed. There is therefore a distinction between the point of reaction and point of decay (or fragment production). At the latter point, the projectile-like nucleus spontaneously decays into two fragments. The initial condition of the two fragments is such that their initial velocity corresponds to an asymptotic relative energy given by $E_{x}$, and their initial position is at their classical turning point for that energy, or their mutual barrier radius, whichever is larger. The fragments and target-like nucleus are then propagated until all nuclei are sufficiently well separated.

The simulations build up a picture of the expected fragment-fragment correlations by Monte Carlo sampling the collision impact parameter, the reaction point, the excitation and lifetime of the intermediate system, and the initial orientation of the breakup fragments. Applications to transfer-triggered breakup through ${ }^{8} \mathrm{Be}[8]$ and direct breakup of ${ }^{6} \mathrm{Li}$ and ${ }^{7} \mathrm{Li}$ [7] have been made, with quite good agreement with experimental observables.

\subsection{Results for ${ }^{7} \mathrm{Li}+{ }^{58} \mathrm{Ni}$}

We now focus on the case of ${ }^{7} \mathrm{Li}+{ }^{58} \mathrm{Ni}$, disintegrating via the proton-pickup breakup mode $(\alpha+\alpha)$ in near-barrier collisions. Experimental data from Ref. [27] and the results of initial calculations are shown in Figure 5. The calculations are filtered by the detector coverage from the experiment such that they can be directly compared with the experimental data. The data is plotted as a function of two angles: $\theta_{12}$, the opening angle of the velocity vectors of the two fragments, and $\beta$, the orientation of their relative motion with respect to the motion of their centre of mass. These angles are illustrated in the bottom left of Figure 5, and give an accessible way to view the fragment correlations, and their sensitivity to target proximity in particular [27]. Distant from the target nucleus, and for a welldefined ${ }^{8} \mathrm{Be}$ excitation energy, there is a distinctive correlation between $\beta$ and $\theta_{12}$. For prompt breakup, this correlation is distorted by the interaction of the $\alpha$-particles and the target. The experimental data [Figure 5(a)] show two distinctive bands - one at $\theta_{12}<15^{\circ}$, and one extending from $\left(\theta_{12}=0^{\circ}, \beta=0^{\circ}\right)$ to $\left(\theta_{12}=70^{\circ}, \beta=90^{\circ}\right)$. The first of these is associated with the ${ }^{8} \mathrm{Be}$ ground state, and follows exactly what one would expect of asymptotic breakup. The second band is associated with prompt breakup of the ${ }^{8} \mathrm{Be}$ $2^{+}$state. For further details, see Ref. [27, 28].

The previous work [27] illustrated the results of the model by focusing on breakup occuring at fixed distances prior to and after the distance of closest approach, without explicitly considering the lifetime of the ${ }^{8} \mathrm{Be}$ state involved. Here we show complete Monte Carlo calculations where the impact parameters, reaction distance, the ${ }^{8} \mathrm{Be}$ excitation energies and lifetimes, and the initial orientation of the $\alpha$ fragments are all stocastically sampled. In Figure 5(d) we show the coordinates at which the reaction was determined to occur $\left(T_{\text {Reaction }}, R_{\text {Reaction }}\right)$ and in Figure $5(\mathrm{e})$ the point at which ${ }^{8} \mathrm{Be}$ decayed into two $\alpha$ particles $\left(T_{\text {Decay }}, R_{\text {Decay }}\right)$. The calculations are consistent with earlier calculations from Ref. [27] which concluded that much of the breakup observed comes from events where the ${ }^{8} \mathrm{Be}$ is receding from the target. Here, however, this is explicitly because the ${ }^{8} \mathrm{Be}$ is allowed to propagate for some time - the duration of which is consistent with the structural properties of the $3 \mathrm{MeV} 2^{+}$state - before decaying into two $\alpha$ particles. Crucially, it seems essential to delay the breakup in order to reproduce the band of events seen.

The agreement with the experimental data is not perfect. One can clearly see an excess of events near $\beta=90^{\circ}$ in Figure 5(c) when compared to the experimental data shown in Figure 5(a). If one compares Figure 5(c) to Figure 5(b) (instantaneous breakup), it suggests that the excess of events is caused from too much breakup occurring near the distance of closest approach, despite the delay of breakup incorporated into the simulations. We discuss some of the possible causes and consequences of this below. 

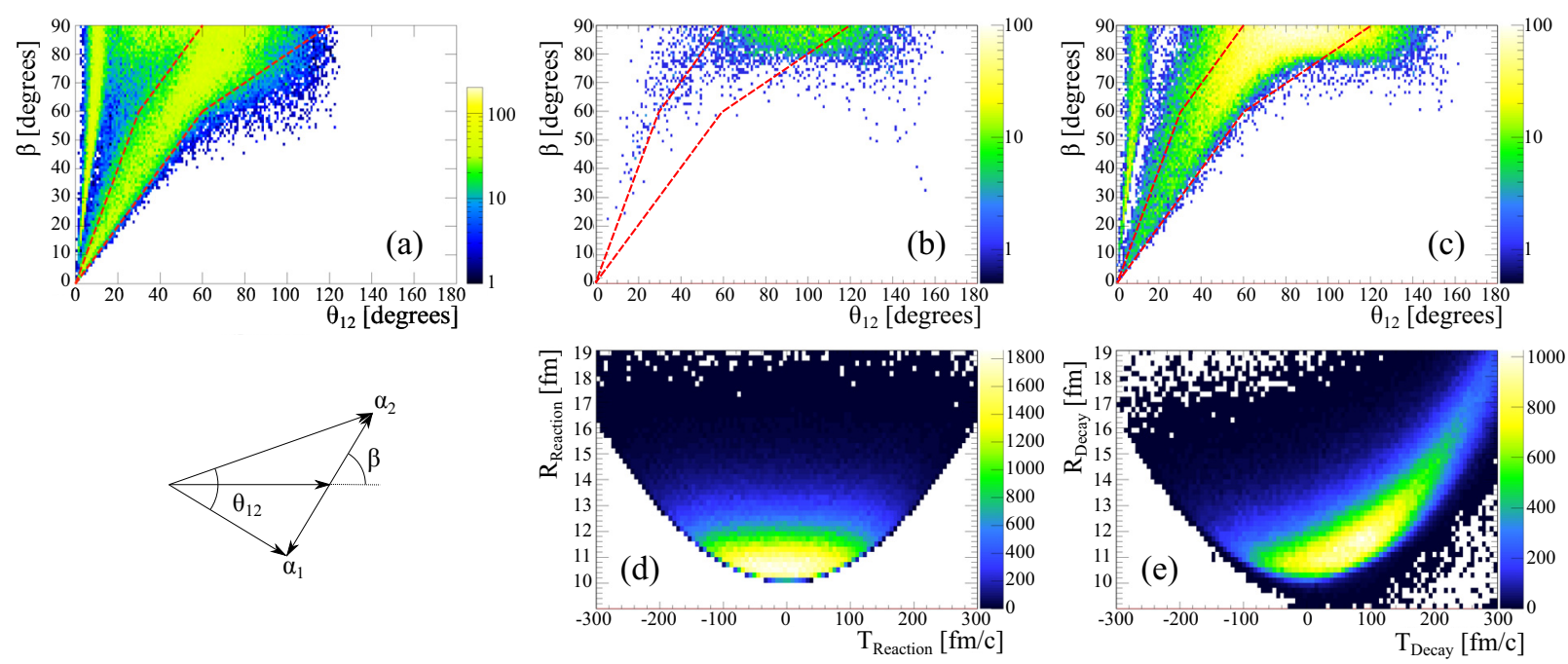

Figure 5. Experimental results and simulations for the ${ }^{7} \mathrm{Li}+{ }^{58} \mathrm{Ni}$ reaction. (a) Experimental data (see Ref. [27] for further details). (b) Simulations assuming that breakup is instantaneous at the point of reaction. (c) Simulations incorporating the lifetime of ${ }^{8} \mathrm{Be}$. (d) Simulated time (with respect to the time of distance of closest approach) and projectile-target distance of the transfer reaction forming ${ }^{8} \mathrm{Be}$. In the simulations (b) where breakup is instantaneous, this also corresponds to the breakup point. (e) Times and positions of breakup for the case where the ${ }^{8} \mathrm{Be}$ lifetime is included. In plots (a-c) red lines are drawn to guide the eye. See text for further discussions.

\section{Discussion and outlook}

As demonstrated by the abundance of talks at this conference, breakup of the weakly-bound stable nuclides ${ }^{6} \mathrm{Li},{ }^{7} \mathrm{Li}$ and ${ }^{9} \mathrm{Be}$, and the relationship between breakup and fusion suppression, is a very active topic of research. Here we have focused on the nature of breakup, and exactly how and why it can influence fusion. The broad conclusion is that each projectile is different, with a variety of mechanisms triggering breakup. Inferring the likely influence on fusion requires a very detailed understanding of the mechanisms triggering the breakup, and on the structure of any intermediate nuclides in particular. We discussed classical dynamical simulations of breakup, focusing on the case of breakup with ${ }^{7} \mathrm{Li}$ projectiles via proton pickup. The simulations indicate that even short-lived resonant states, such as the ${ }^{8} \mathrm{Be} 2^{+}$, may survive for a sufficiently long time so as to not be able to influence fusion significantly at above barrier energies.

The results also hint that there is some stabilisation of ${ }^{8} \mathrm{Be}$ that prevents it breaking up when near the distance of closest approach. The nature of this, and the cause is unclear. It may be that the present structure model for ${ }^{8} \mathrm{Be}$ is not good enough, or that the Coulomb field of the target changes the structure of ${ }^{8} \mathrm{Be}$, possibly causing it to be stabilised. A similar phenomenon has been hinted at in ${ }^{8} \mathrm{Be}$ emission following heavy-ion collisions [29].

Another possible cause of disagreement could be polarization of the ${ }^{7} \mathrm{Li}$ cluster projectile by its interaction with the target. Classically, the different charge to mass ratios of the two intrinsic clusters ( $\alpha$ and triton) could cause them to orient themselves with respect to the Coulomb field. This may have some impact on the observed distribution of events with $\beta$ and $\theta_{12}$ A related question would be that of tidal effects once the reaction triggering breakup has occurred, but prior to production of the breakup fragments. In the present purely classical approach the intermediate resonance state is treated as immutable until it decays into two fragments. For narrow states, this is perhaps a reasonable approximation, but it is less clear that broad and non-resonant states - i.e. those contributing to the prompt breakup — will be similarly unaffected.

It is not possible to sensibly answer all these questions within the scope of classical dynamical models, which are inherently limited by their reliance on classical mechanics, and cannot predict the strength of these various effects. Classical dynamical models can, however, give some idea of the likely impacts of these effects on the breakup fragment correlations and on above-barrier complete and incomplete fusion. What is clear is that we must go beyond identifying those cases where fusion suppression occurs, and develop a more sophisticated understanding of the near-barrier breakup of weakly-bound nuclides. In nearfuture reaction studies with dripline nuclides, this will be absolutely essential.

\section{Acknowledgements}

This work was supported by Australian Research Council Grant Nos. FT120100760 and DP170102423.

\section{References}

[1] J.R. Leigh, M. Dasgupta, D.J. Hinde, J.C. Mein, C.R. Morton, R.C. Lemmon, J.P. Lestone, J.O. Newton, H. Timmers, J.X. Wei et al., Phys. Rev. C 52, 3151 (1995)

[2] G. Audi, M. Wang, A. Wapstra, F. Kondev, M. MacCormick, X. Xu, B. Pfeiffer, Chinese Physics C 36, 1287 (2012) 
[3] M. Dasgupta, D.J. Hinde, K. Hagino, S.B. Moraes, P.R.S. Gomes, R.M. Anjos, R.D. Butt, A.C. Berriman, N. Carlin, C.R. Morton et al., Physical Review C 66, 041602(R) (2002)

[4] M. Dasgupta, P.R.S. Gomes, D.J. Hinde, S.B. Moraes, R. M.Anjos, A.C. Berriman, R.D. Butt, N. Carlin, J. Lubian, C.R. Morton et al., Physical Review C 70, 024606 (2004)

[5] L.F. Canto, P.R.S. Gomes, R. Donangelo, J. Lubian, M.S. Hussein, Physics Reports 596, 105 (2015)

[6] E.F. Aguilera, E. Martinez-Quiroz, D. Lizcano, A. Gómez-Camacho, J.J. Kolata, L.O. Lamm, V. Guimarães, R. Lichtenthäler, O. Camargo, F.D. Becchetti et al., Phys. Rev. C 79, 021601 (2009)

[7] S. Kalkal, E.C. Simpson, Physical Review C 93, 044605 (2016)

[8] K.J. Cook, E.C. Simpson, D.H. Luong, S. Kalkal, M. Dasgupta, D.J. Hinde, Phys. Rev. C 93, 064604 (2016)

[9] S.P. Hu, G.L. Zhang, J.C. Yang, H.Q. Zhang, P.R.S. Gomes, J. Lubian, J.L. Ferreira, X.G. Wu, J. Zhong, C.Y. He et al., Phys. Rev. C 93, 014621 (2016)

[10] V.V. Parkar, V. Jha, S. Kailas, Phys. Rev. C 94, 024609 (2016)

[11] E.F. Aguilera, P. Amador-Valenzuela, E. MartinezQuiroz, J. Fernández-Arnáiz, J.J. Kolata, V. Guimarães, Phys. Rev. C 93, 034613 (2016)

[12] V. Pesudo, M.J.G. Borge, A.M. Moro, J.A. Lay, E. Nácher, J. Gómez-Camacho, O. Tengblad, L. Acosta, M. Alcorta, M.A.G. Alvarez et al., Phys. Rev. Lett. 118, 152502 (2017)

[13] K. Vo-Phuoc, C. Simenel, E.C. Simpson, Phys. Rev. C 94, 024612 (2016)

[14] M. Fisichella, A.C. Shotter, A. Di Pietro, P. Figuera, M. Lattuada, C. Marchetta, V. Privitera, L. Romano, C. Ruiz, M. Zadro, Phys. Rev. C 92, 064611 (2015)

[15] M. Fisichella, A.C. Shotter, P. Figuera, J. Lubian, A. Di Pietro, J.P. Fernandez-Garcia, J.L. Ferreira, M. Lattuada, P. Lotti, A. Musumarra et al., Phys. Rev.
C 95, 034617 (2017)

[16] R. Rafiei, R. du Rietz, D.H. Luong, D.J. Hinde, M. Dasgupta, M. Evers, A. Diaz-Torres, Phys. Rev. C 81, 024601 (2010)

[17] D.H. Luong, M. Dasgupta, D.J. Hinde, R. du Rietz, R. Rafiei, C.J. Lin, M. Evers, A. Diaz-Torres, Physics Letters B 695, 105 (2011)

[18] D.H. Luong, M. Dasgupta, D.J. Hinde, R. du Rietz, R. Rafiei, C.J. Lin, M. Evers, A. Diaz-Torres, Physical Review C 88, 034609 (2013)

[19] L.R. Gasques, D.J. Hinde, M. Dasgupta, A. Mukherjee, R.G. Thomas, Physical Review C 79, 034605 (2009)

[20] B. Wang, W.J. Zhao, P.R.S. Gomes, E.G. Zhao, S.G. Zhou, Physical Review C 90, 034612 (2014)

[21] A. Diaz-Torres, D.J. Hinde, J.A. Tostevin, M. Dasgupta, L.R. Gasques, Physical Review Letters 98, 152701 (2007)

[22] A. Diaz-Torres, Computer Physics Communications 182, 1100 (2011)

[23] L.C. Chamon, D. Pereira, M.S. Hussein, M.A. Cândido Ribeiro, D. Galetti, Phys. Rev. Lett. 79, 5218 (1997)

[24] D.J. Hinde, M. Dasgupta, B.R. Fulton, C.R. Morton, R.J. Wooliscroft, A.C. Berriman, K. Hagino, Phys. Rev. Lett. 89, 272701 (2002)

[25] A.M. Lane, R.G. Thomas, Reviews of Modern Physics 30, 257 (1958)

[26] F.C. Barker, Australian Journal of Physics 41, 743 (1988)

[27] E.C. Simpson, K.J. Cook, D.H. Luong, S. Kalkal, I.P. Carter, M. Dasgupta, D.J. Hinde, E. Williams, Physical Review C 93, 024605 (2016)

[28] E.C. Simpson, K.J. Cook, M. Dasgupta, S. Kalkal, D.H. Luong, I.P. Carter, D.J. Hinde, E. Williams, EPJ Web Conf. 123, 03002 (2016)

[29] A.B. McIntosh, S. Hudan, C.J. Metelko, R.T. de Souza, R.J. Charity, L.G. Sobotka, W.G. Lynch, M.B. Tsang, Phys. Rev. Lett. 99, 132701 (2007) 